\title{
Endometriosis y Factor Tubo Peritoneal
}

\author{
Dres.: Jaime Ferro Camargo*, Germán Montoya Sánchez**
}

La Endometriosis es una de las enfermedades que altera multifactorialmente la fertilidad de la mujer $y$ es concomitantemente con la enfermedad pélvica inflamatoria, las entidades que más alteran el factor Tubo Peritoneal. Es bueno recordar que la intensidad de las manifestaciones clínicas de la enfermedad no tienen relación directa con el grado de alteración que ocasionen las lesiones en la pélvis, de tal forma que una pequeña lesión puede no dar ringuna sintomatología específica en una paciente, mientras en otra, puede producir tales manifestaciones sistomáticas como las que produciría una lesión muchísimo mayor. En forma general la exploración física puede ser positiva en la medida que las alteraciones sean más aparentes o las lesiones sean mayores; pero la impresión diagnós-

* Instructor. Departamento de Ginecología y Obstetricia. Unidad de Endoscopia Ginecológica. Hospital de San José. Facultad de Medicina del Rosario.

** Jefe del Departamento de Ginecología y Obstetricia. Hospital de San José. Facultad de Medicina del Rosario. tica debe ser siempre certificada mediante la práctica de exámenes complementarios especialmente cuando se refiere al estudio de los casos que comprometen la fertilidad.

En los casos más leves de la enfermedad la alteración de la fertilidad generalmente nos es debida a una alteración morfológica sino a mecanismos inmuno - bioquímico - hormonales que alteran la motilidad normal de las trompas uterinas, la adecuación del endometrio normal o provocan la falta de ovulación por la luteinización del folículo no roto caracterizado por el Síndrome de L.U.F. Pero en grados más avanzados se observa la encarcelación de los ovarios entre adherencias quedando perdidos los óvulos entre estas, o el impedimento de la captación ovular por las fimbrias tubáricas debido a fijación de las trompas uterinas por adherencias de diferentes consistencias, configuraciones, extensiones y localizaciones que en ningún momento al teran o dan indicios concluyentes en las pruebas de permeabilidad tubárica como el Test de Rubin o pueden ser evidenciadas mediante el ultrasonido pélvico o sólo en 
algunos pocos casos excepcionalmente la Histerosalpingografía puede mostrar remanentes de medio de contraste en forma tabicada o escalonada en la pelvis. De tal forma que hoy en día es innegable que no sólo el diagnóstico de precisión, sino la clasificación adecuada, manejo, pronóstico y control de Endometriosis se hace a través de la Laparoscopia.

Es por eso que en nuestro presente estudio revisamos estadísticamente un gran número de casos, lo cual nos autoriza el poder hablar en forma contundente de la incidencia de la Endometriosis en nuestro medio, de la incidencia de la enfermedad de acuerdo con la intensidad y severidad de las lesiones, a la incidencia de las alteraciones tubo - peritoneales como hallazgo laparoscópico, a las relaciones que existen entre la Endometriosis y la alteración del Factor Tubo Peritoneal, a la incidencia de alteración tubo peritoneal causada por Endometriosis y la gravedad de las lesiones y modificaciones producidas en la pelvis, dadas por una clasificación novedosa que hemos implementado y puesto en práctica la cual nos permite una correlación directa de los grados con las alteraciones pélvicas y el pronóstico de fertilidad. Además, mediante la modificación del último paso de la Terapia de los Tres Pasos: laparoscopia, hormonoterapia y el último, laparoscopia control, por el de microcirugía para cirugía conservadora especialmente destinada a los casos con alteración del Factor Tubo Peritoneal, para poder encausar más adecuadamente el manejo y seguimiento de las pacientes.

\section{MATERIAL Y METODOS}

Se llevo a cabo una revisión estadística de las laparoscopias practicadas en cinco años desde 1980 hasta 1984 inclusive en la Unidad de Endoscopia Ginecológica del Departamento de Ginecología y Obstetricia del Hospital de San José, Facultad de Medicina del Colegio Mayor de Nuestra Señora del Rosario y cuyo diagnostico resultó ser Endometriosis. Esta había sido de antemano clasificada en cuatro grados a saber: leve, moderada, severa y extensa siguiendo los parámetros de clasificación dados por la Sociedad Americana de Fertilidad y Esterilidad (A.F.S.) en 1979. (Gráfica 1). y los cuales están representados por la suma de puntajes dados según el tamaño de las lesiones, ya sea área de las placas o volumen de los nódulos y quistes; de la localización en peritoneo, ligamentos uterosacros, ovarios y trompas uterinas además, califica no sólo las lesiones sino también las adherencias de acuerdo a que correspondan a formaciones finas o transparentes, densas o extensas tanto como en el sitio en que se encuentren llegando a encarcelar y obliterar los órganos femeninos internos.

En cuanto a la alteración del factor Tubo Peritoneal también se reviso estadisticamente tanto en forma independiente como en relación concomitante con la Endometriosis y se estudio más detenidamente la alteración que produce la Endometriosis en cuanto al Factor Tubo' Peritoneal y se utilizó para el efecto una clasificación de alteración implementada por nosotros en el servicio y la cual nos ha servido no sólo para la valoración de los diferentes casos sino también para pronóstico y evaluación de resultados. Esta se divide en tres grados de calificación: (Gráfica No. 2). Leve, Moderada y Severa alteración del factor Tubo Peri. toneal dados por las modificaciones que las adherencias o lesiones de la enfermedad base, en este caso la Endometriosis, ocasionen en la permeabilidad tubárica o 
GRAFICA No. 1

\section{PROTOCOLO DE ENDOMETRIOSIS}

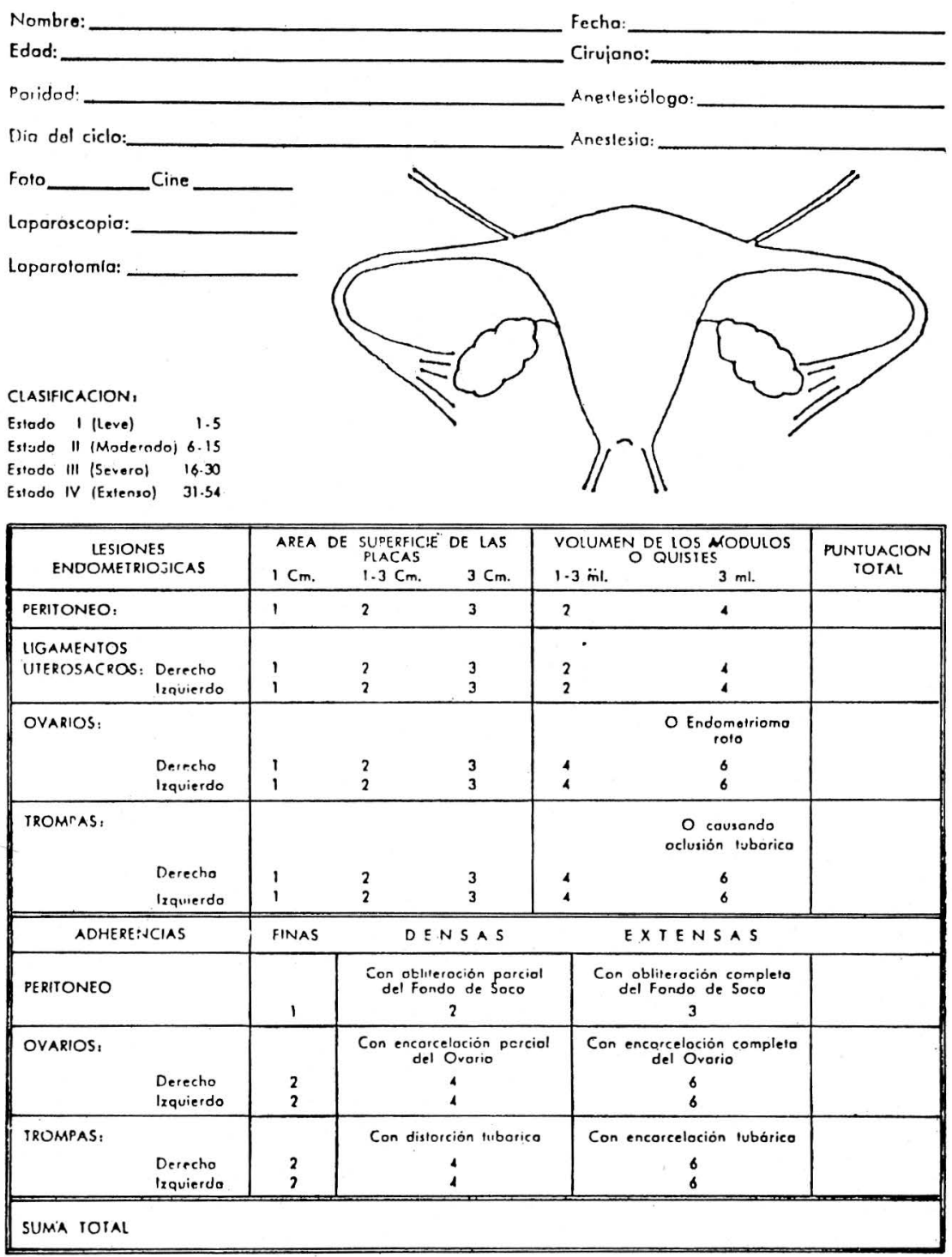

Tomado y traducido de la Revista de Fertilidad y Esterilidad de la Sociedad Americana de Fertilidad, 1979. 
en la no encarcelación o encarcelación parcial uni o bilateral o la encarcelación completa de los ovarios de tal forma que se refiere por completo a la prognosis de fertilidad en la paciente.

GRAFICA No. 2

FACTOR TUBO - PERITONEAL

\begin{tabular}{|c|c|c|c|}
\hline \multicolumn{4}{|c|}{ FACTOR TIIBO - PERI TONEAL } \\
\hline F.T.P. & $\begin{array}{l}\text { SIN } \\
\text { ENCARCELAQON }\end{array}$ & $\begin{array}{l}\text { ENCARCELACION } \\
\text { INILATEPAL }\end{array}$ & $\begin{array}{l}\text { ENCARCELACION } \\
\text { BILATEPAL }\end{array}$ \\
\hline $\begin{array}{l}\text { PEPPEABILIDAD } \\
\text { BILATERAL }\end{array}$ & LEVE & MODERADA & SEVERA \\
\hline $\begin{array}{l}\text { PEPPEABILIDAD } \\
\text { WILATEPAL }\end{array}$ & MODERADA & MODERADA & SEVERA \\
\hline $\begin{array}{c}\text { SIN } \\
\text { PEPEEABILIDAU }\end{array}$ & SEVERA & SEVERA & SEVERA \\
\hline
\end{tabular}

Una vez unificados y establecidos los criterios de clasificación del problema se procedió a la tabulación y diagramación de los diferentes hallazgos pudiendo establecer y relacionar datos bastante interesantes como la incidencia de Endometriosis como entidad y por clasificación de grados de severidad que ocurre entre las pacientes que coricurrieron al Hospital por estudio de Fertilidad y Dolor Pélvico sumados y que requirieron laparoscopia; en este estudio no se hizo discriminación por motivo de consulta ya que esto fue analizado en otro trabajo preliminar hace dos años y en el actual el objetivo principal corresponde a los datos que relacionan la alteración de las estructuras pélvicas dependientes de las lesiones Endometriósicas. De manera que se estableció la incidencia del Factor Tubo Peritoneal tanto en forma independiente como en relación con el grado de severidad de alteración de acuerdo con nuestra clasificación ya explicada y en forma dependiente la Endometriosis en situación incidental y en relación con intensidad gradual de la enfermedad: pudiendo establecer conclusiones bastante directas e informativas con respecto a la Fertilidad de las pacientes comprometidas por diferentes grados de la enfermedad. Además, no podemos dejar de lado explicar el manejo que hemos venido dando a las pacientes en nuestro servicio y que corresponde a la Terapia de los Tres Pasos y que hemos tomado de la llevada a cabo por el Profesor Kurt Semm de Kiel, Alemania en 1978 y promulgada en junio de 1982 en el Congreso Mundial de Fertilidad y Esterilidad en Dublin, Irlanda y que gentilmente hemos modificado o acondicionado en su último paso como claramente lo referiremos en la siguiente parte de Técnica y cuya realización esta íntimamente ligada al manejo del Factor Tubo Peritoneal alterado por Endometriosis.

\section{TECNICA}

Como marco para describir las Técnicas empleadas desarrollaremos el tema con base en los Tres Pasos de la Terapia empleada en el manejo del problema de las pacientes afectadas. Clásicamente la Terapia de los Tres Pasos corresponde a un primer paso Laparoscópico, un segundo paso de Hormonoterapia y un tercer y último paso en el cual se practica una nueva Laparoscopia, pero es aquí donde lo modificamos pues en su lugar practicamos, una Laparotomia Ginecológica para procedimientos Microquirúrgicos. Para iniciar el desarrollo de cada uno de los pasos comenzaremos mencionando que no todos son necesariamente aplicados a todas las pacientes, pues de acuerdo con el grado de alteración en cada una de ellas será necesario el uso de sólo el pri- 
mero como solución única o de todos los pasos para lograr la completa remisión del problema.

En referencia al primer paso usamos la Laparoscopia no sólo como fín diagnóstico sino como implemento operativo y por esto empleamos en todos los casos anestesia general y por punción abdominal a nivel periumbilical usando aguja de Verres practicamos el neumoperitoneo con NO2 usando volumenes variables de acuerdo con una presión media de seguridad que no excede los $16 \mathrm{~mm} \mathrm{Hg}$. en el indicador del Celiometro. Luego por incisión periumbilical no mayor de un centímetro se pasa un trocar con su camisa de $11 \mathrm{~mm}$. de diámetro con válvula para gas a mantenimiento $y$ a través de esta camisa se pasa un telescopio de $10 \mathrm{~mm}$. de diámetro con lente $0^{\circ}$ y canal operatorio de $6 \mathrm{~mm}$. de diámetro y con transmisión de luz fría a través de cable de fibra de vidrio de una fuente de luz con capacidad para dar desde 125 hasta 550 watios y con sistema de flash para fotografía a color.

Además, se practica una segunda punción Laparoscópica a nivel suprapúbico pudiendo ser medial o lateral según la necesidad y de $0.5 \mathrm{cms}$. por donde se pasa un trocar con su camisa de $5 \mathrm{~mm}$. para el paso de pinzas operatorias especialme?te el paso de la punta del Endocuagulador o de la pinza caimán para Endocuagulación de las lesiones especialmente de la) adherencias y posterior paso de tijeras para el corte y liberación de estas; el paso de diferentes agujas para punción y drenaje de quistes; el paso de diferentes pinzas atraumáticas de prensión para enucleación de quistes, resección de adherencias, dilatación de Fimbrias Tubáricas y manipulación de todas las estructuras involucradas y que permitan sin mayores riesgos y de acuerdo con el grado de severidad el manejo inicial o completo de la entidad en este paso. (Fig. No. 1).

FIGURA No. 1

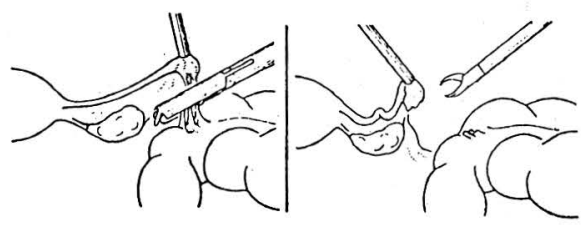

Vale la pena hacer una especial mención del citado sistema de endocuagulación, que hasta cierto punto viene a ser novedoso frente a otros sistemas. De forma común se venía usando corriente de alta frecuencia (H.F.) en sistemas unipolares o bipolares que básicamente consisten en el paso de la corriente a través de los tejidos interpuestos entre un electrodo $y$ una placa $o$ entre un electrodo $y$ otro de tal forma que a través de los líquidos corporales y substancias electrolíticamente conductoras se produce una resistencia que aumenta la temperatura y cauteriza el tejido. (Fig. No. 2).

FIGURA No. 2

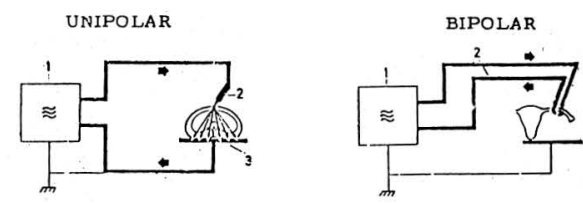

1: GENERADOR, 2: ELECTRODOS, 3: PLACA

En cambio el sistema de endocuagulación se basa en el uso de corriente de bajo voltaje, la cual por medio del calentamiento de la potente resistencia interna de las puntas de las pinzas, transmiten un calor regulado en intensidad y tiempo codificado de antemano en el comando del aparato y siendo bastante limitada la "quemadura" sobre el tejido, sin que 
exista paso de corriente produciendo una especie de cocimiento del tejido por desecación y cuagulación de las proteínas, independiente del contenido electrolítico. (Fig. No. 3). Esto aumenta en una forma bastante amplia la seguridad en el uso de sistemas biofísicos para la necrosis de tejidos mediante métodos endoscópicos.

FIGURA No. 3

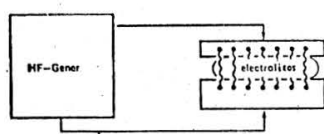

(1) alta' faecuencia
(2) EnOOCUAGULACION

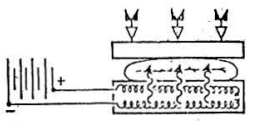

Para finalizar el procedimiento Laparoscópico que veníamos describiendo, extraemos del abdomen la mayor cantidad de gas posible y colocamos un punto subdermico a nivel de las incisiones prácticadas con materiales absorbibles.

Con respecto al segundo paso terapéutico que corresponde al de hormonoterapia sólo queremos referir que básicamente se han empleado en la actualidad dos tipos diferentes de medicamentos, uno el Sulfato de Danazol, poco usado en nuestro medio más que todo debido al alto costo comercial y el otro, el Acetato de Medroxi-progesterona, más adecuadamente por vía oral para la regluación de la dosis, que fluctua entre veinte a cuarenta mgrs. diarios con duración de tres, seis, nueve meses o hasta un año de acuerdo con la clasificación de severidad de la Endometriosis y a la respuesta al tratamiento.

En el tercer paso terapéutico, en el que clásicamente se practicaba una nueva Laparoscopia con el fin de controlar el resultado de la Hormonoterapia y además realizar por medio de esta un procedimiento operatorio en forma complementaria, lo hemos modificado ya que no en todas es factible esta práctica, de manera que en nuestro servicio resulta infrecuente y ocasional en algunos estados moderados y casi siempre resultamos practicando una Laparotomía Ginecológica para que con técnicas Microquirúrgicas se realice con mayor precisión la resección completa de las lesiones endometriosicas residuales y lo que es más fundamental en la terapia de los grados, más avanzados de la enfermedad correspondiente al manejo de las severas adherencias que modifican y alteran las diferentes estructuras pélvicas comprometidas y además, utilizar todos los recursos técnicos para evitar nuevamente la aparición de estas.

\section{RESULTADOS}

Desde 1980 hasta 1984 inclusive, realizamos en la Unidad de Endoscopia Ginecológica del Hospital de San José un total de 534 Laparoscopias, las cuales habían sido practicadas básicamente y en su orden por: Dolor Pélvico, Estudios de Fertilidad y Masa Pélvica. En la revisión encontramos que de todas estas Endoscopias, noventa y dos correspondían a Endometriosis, lo cual significa una incidencia del $17.2 \%$ cifra más o menos bien correlacionada con lo encontrado en estudios similares por otros autores. Si observamos más detalladamente el cuadro No. 1 podemos darnos cuenta que la incidencia mostró un aumento hacia el tercer y cuarto año de la revisión, para luego estabilizarce nuevamente y como lo habíamos referido en un estudio preliminar, esto posiblemente se debía a una mejor implementación técnica y a un diferente enfoque del problema. 
CUADRO No. 1

INCIDENCIA ENDOMETRIOSIS

LAPAROSCOPIA 1980 - 1984 HOSPITAL DE SAN JOSE

\begin{tabular}{|c|c|c|c|}
\hline ANO & LAPAROSCOPIAS & ENDOMETRIOSIS & $\%$ \\
\hline 1980 & 94 & 8 & 8.5 \\
\hline 1981 & 94 & 9 & 9,6 \\
\hline 1982 & 108 & 16 & 14,8 \\
\hline 1983 & 103 & 25 & 24,3 \\
\hline 1934 & 135 & 34 & 25,2 \\
\hline & 534 & 92 & 17,2 \\
\hline
\end{tabular}

En el cuadro No. 2 se detalla la incidencia de la Endometriosis por grados de severidad de acuerdo con los cuatro parámetros dados por la clasificación de la Sociedad Americana de Fertilidad y Esterilidad (A.F.S.) y claramente encontramos que más del $60 \%$ de la enfermedad corresponden a los grados menos avanzados de la entidad es decir, al leve más el moderado, pero si tenemos en cuenta sólo la alteración leve, esta corresponde al $40.2 \%$ cifra que hay que tener muy en cuenta cuando la comparemos con la incidencia de alteración del factor Tubo Peritoneal por grados de Endometriosis.

CUADRO No. 2

\section{CLASIFICACION ENDOMETRIOSIS}

ANO LEVE MODERADA SEVERA EXTENSA

$\begin{array}{ccccc}1980 & 4 & 3 & 1 & - \\ 1981 & 3 & 3 & 2 & 1 \\ 1982 & 7 & 3 & 2 & 4 \\ 1983 & 9 & 6 & 5 & 5 \\ 1984 & \frac{14}{37} & \frac{9}{24} & \frac{7}{16} & \frac{4}{14} \\ \text { TOTAL } & 37 & 16.4 & 15.2 \\ \% & 40.2 & 26.0 & 17.4\end{array}$

Con respecto a la incidencia del Factor Tubo Peritoneal y revisado en forma independiente de la entidad que lo estuviera produciendo, podemos ver en el cuadro No. 3 que de las 534 Laparoscopias prácticadas 238 o sea el $44.6 \%$ lo mostraron alterado independientemente del grado de severidad y en el cuadro No. 4 y con una distribución de proporciones semejantes en los diferentes años analizados, encontramos que el $58.8 \%$ corresponden a una alteración severa del Factor Tubo Peritoneal lo cual nos hace pensar que según nuestra clasificación hay un grave compromiso de la Fertilidad de estas pacientes.

CUADRO No. 3

\section{INCIDENCIA DEL FACTOR TUBO PERITONEAL}

$\begin{array}{cccc}\text { ANO } & \text { LAPAROSCOPIAS } & \text { FAC. TUBO-PERITONEAL } & \% \\ 1980 & 94 & 41 & 43,6 \\ 1981 & 94 & 36 & 38,3 \\ 1982 & 108 & 53 & 49,1 \\ 1983 & 103 & 49 & 47,6 \\ 1984 & \underline{135} & \underline{59} & \frac{43,7}{44,6}\end{array}$

CUADRO No. 4

\section{CLASIFICACION DEL FACTOR TUBO PERITONEAL}

$\begin{array}{ccccc}\text { ANO } & \text { LEVE } & \text { MODERADA } & \text { SEVERA } & \text { TOTAL } \\ 1980 & 8 & 12 & 21 & 41 \\ 1981 & 5 & 14 & 17 & 36 \\ 1982 & 7 & 15 & 31 & 53 \\ 1983 & 8 & 8 & 33 & 49 \\ 1984 & 6 & 15 & 38 & 59 \\ \text { TOTAL } & 34 & 64 & 140 & 238 \\ \% & 14,3 & 26,9 & 58,8 & 44,6\end{array}$

Con respecto a la Endometriosis y a la alteración del Factor Tubo Peritoneal encontramos según el cuadro No. 5, que 
CUADRO No. 5

\section{RELACION INCIDENCIA \\ ENTRE ENDOMETRIOSIS Y \\ ENDOMETRIOSIS CON FACTOR \\ TUBO-PERITONEAL}

ANO

LAPAROSCOPIAS ENDOMETRIOSIS

ENDOMET, + F.T,P $\%$

$\begin{array}{lrrrl}1980 & 94 & 8 & 4 & 50 \\ 1981 & 94 & 9 & 6 & 67 \\ 1982 & 108 & 16 & 8 & 50 \\ 1983 & 103 & 25 & 16 & 64 \\ 1984 & \frac{135}{534} & \frac{34}{92} & \frac{18}{52} & \frac{53}{56.5}\end{array}$

el $56.5 \%$ de las pacientes con Endometriosis en la presente revisión tenían alteración del Factor, siendo una cifra que más o menos se venía manteniendo con muy ligeras fluctuaciones a través de los años analizados y que afortunadamente y según el cuadro No. 6 la incidencia de tal alteración no se encontró en los casos leves que son más frecuentes y los de más fácil manejo y para los casos más complicados como son los de severa y extensa alteración se encontró un compromiso del Factor en un 57.7\% lo cual hace que el manejo de la entidad requiera invariablemente la implementación del

CUADRO No. 6

INCIDENCIA GENERAL

Y POR CLASIFICACION DE

ENDOMETRIOSIS CON FACTOR

TUBO-PERITONEAL

$\begin{array}{cccccc}\text { ANO } & \text { LEVE } & \text { MODERADA } & \text { SEVERA } & \text { EXTENSA } & \text { TOTAL } \\ 1980 & - & 3 & 1 & - & 4 \\ 1981 & - & 3 & 2 & 1 & 6 \\ 1982 & - & 2 & 2 & 4 & 8 \\ 1983 & - & 6 & 5 & 5 & 16 \\ 1984 & - & 8 & 6 & \frac{4}{16} & \frac{18}{52} \\ \text { TOTAL } & - & 22 & 16 & 14 & 52,9 \\ \% & - & 42,3 & 30,8 & 26,9\end{array}$

último paso de la terapia modificada que hemos expuesto en el presente trabajo.

\section{CONCLUSIONES}

Según se desprende de los hallazgos consignados de los cuadros anteriores y de lo que podemos observar en las gráficas 3 y 4 casi la mitad de los casos que llegan a Laparoscopia en nuestra Unidad presentan alteraciones del Factor Tubo Peritoneal sin hacer referencia a la patología de base. Más de la mitad de los casos con Endometriosis presentan alteración del Factor Tubo Peritoneal y de estos más de la mitad presentan alteraciones severas según nuestra clasificación y tan sólo los casos leves de Endometrio-

GRAFICA No. 3

\section{RELACION ENTRE ENDOMETRIOSIS \\ Y FACTOR TUBO PERITONEAL}

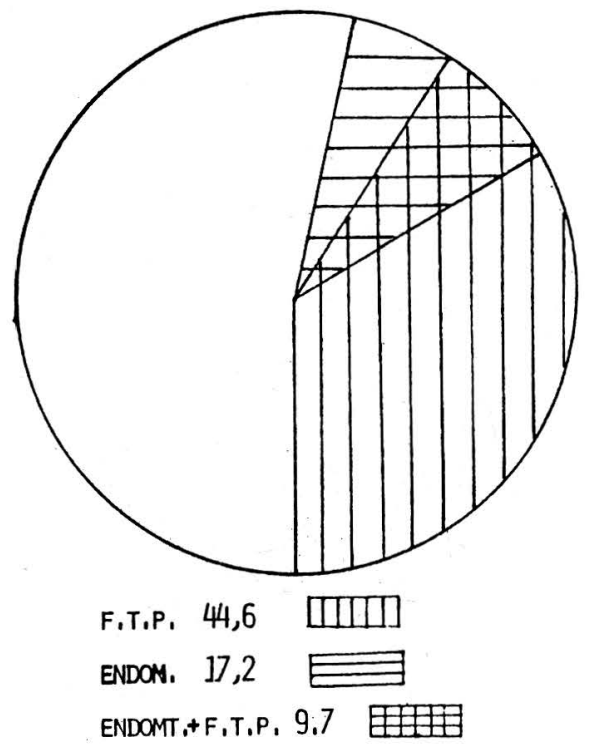


sis no presentan ningún tipo de alteración Tubo Peritoneal.

\section{GRAFICA No. 4 \\ INCIDENCIA ENDOMETRIOSIS CON FACTOR TUBO-PERITONEAL}

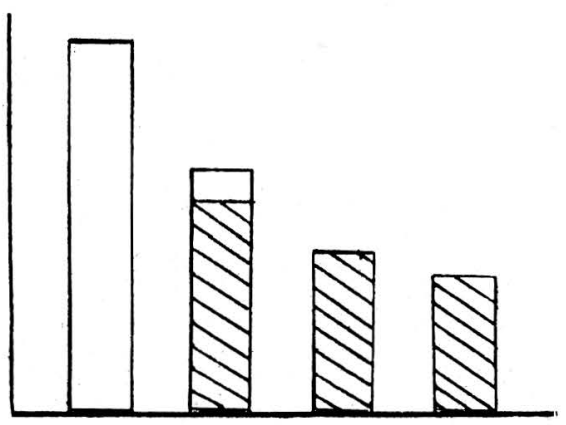

SIN FACTOR TUBO PERITONEAL

CON FACTOR TUBO PERITONEAL

\section{BIBLIOGRAFIA}

1. ANDREWS, W.C., y LARSEN, G.D., 1974 Endometriosis: Tratamiento de Seudoembarazo hormonal $y / 0$ cirugía. Am. J. Obstet. Gynaec. 118, 643.

2. BOECKX, W., GORDTS. S., BROSENS, I. 1980. Microcirugía ovárica en Microcirugía de mujeres Infertiles (Ed) Crosignani P.G. y Rubin B.C., London, Academic Press p.p. 59-76.

3. BONNAR J. 1983. Manifestaciones clínicas y diagnósticos de Endometriosis. Irish J ournal of Medical Science 1952: 5.

4. BROSENS, I.A., KONINCKX. y CORVELEYN, P.A. 1978. Un estudio de Progesterona, 17 B-Estradiol, Prolactina en plasma y niveles de L.H. en la fase lútea y apariencia de los ovarios en pacientes con Endometriosis. Brit. J. Obstet. Gynaecol 85, 246.
Esto nos hace concluir que la Endometriosis al constituirse como la quinta causa más frecuente de alteración de la fertilidad y que teniendo una incidencia de alteración multifactorial especialmente lo que hace referencia al Factor Tubo Peritoneal en más de las tres quintas partes de las pacientes que la padecen y siendo esta alteración uno de los mayores problemas en el manejo de la recuperación de la fertilidad, esta seguirá siendo un reto más para el Ginecólogo de hoy a pesar de la implementación de una excelente tecnología, de un enfoque que implique, no el hallazgo ocasional de la entidad sino la busqueda rutinaria y sistemática y la aplicación de la metodología Terapéutica de los tres pasos que hoy en día parece ser una de las más adecuadas y razonables si lo hacemos en forma escalonada y de acuerdo con la intensidad y severidad de cada caso en particular.

5. BUTTRAN VC Jr: Cirugía conservadora para endometriosis en la mujer fertil. Steril 31: 117, 1977.

6. CZERNOBLISKY, B. y SILVERSTEIN, A. 1978 Salpingitis $y$ Endometriosis ovárica. Fertil Steril. 30, 45.

7. DANIEL, J.F., CHRISTIANSON, C., 1981. Laparoscopia operatoria combinada con terapia con Danazol para endometriosis pélvica. Fertil. Steril 35: 5, 521.

8. DIZEREGA, G.S., BARBER, D.L. y HOOGEN, G.D. 1980. Endometriosis: Rol de los esteroides ováricos en la iniciación, mantenimiento y supresión Fertil. Steril. 33, 649.

9. DMOWSKI, W.P., RAO, R. y SCOMMEGNA, A. 1980. El Síndrome de Folículo Luteinizado no roto y Endometriosis. Fertil. Steril 33, 30. 
10. DMOWSKI, W.P., STEELE, R.W. Y BAKER, G.F. 1981. Deficiente inmunidad celular en Endometriosis. Amer. J. Obs. tet. Gynecol. 141, 377.

11. GREENBLATT, R.B., TZINGOUNIS, V. 1979. Tratamiento de Endometriosis con Danazol. Fertil. Steril 32: 5, 518.

12. KISTNER, R.W. 1975. Manejo de Endometriosis en pacientes infértiles. Fertil Steril. 26, 1151.

13. KISTNER RW: 1979, Endometriosis y Fertilidad. Clin Obstet Gynecol 22: 101, March.

14. KONINCKX, P.R., IDE, P., VANDENBROUCKE, W., .BROSENS, I.A., 1980, nuevos aspectos en la patofisiología de Endometriosis asociada a Infertilidad. J. Reprod. ed. 24, 257-260.

15. METTLER, L. y SEMM, K., 1979. Experiencias clínicas y bioclínicas con Danazol en el tratamiento de Endometriosis en casos de mujeres infertiles. Postgraduate Medical Journal 55: (Supl. 6). 27-32.

16. METTLER, L. Y SEMM, K. 1983. Tres pasos Medicoquirúrgicos en el tratamiento de la Endometriosis. Irish J., med. Science 152: sup. 2.
17. REIVA, H.L., WILSON, J.H. y KAWASAKI, D.M., 1961. Efectos del Noretinodrel en Endometriosis. Am. J. Obstet. Gynec. 82: 109.

18. SAMPSOM, J.A. 1921. Quistes perforados hemorrágicos del ovario. Arch. Surg. 3,245 .

19. SEMM K. 1977. Atlas de Laparoscopia Ginecológica e Histeroscopia Saunders.

20. Sociedad Americana de Fertilidad, 1979. Clasificación de Endometriosis. Fertil Steril 32: 633.

21. SOULES, M.R., MALINAK, L.R., BURY, R. Y POINDEXTER, A. 1976. Endometriosis y anovulación: un problema que coexiste en la mujer infértil. Amer. J. Obstet. Gynecol. 125, 412.

22. STRATHY J.H., 1982: Endometriosis e Infertilidad un estudio laparoscópico de Endometriosis entre mujeres fértiles e infértiles. Fertil Steril 38: 667.

23. WILLIAM, T.J. PRATT S.H: Endome-triosis en 1000 Celiotomias consecutivas: incidencia y manejo. Am. J. Obstet Gynecol.

24. WILLIAMS, E.A. 1983. Aspectos quirúrgicos en la práctica clínica de la Endometriosis. Irish J. Med. Science 152 , sup. 2. 